\title{
The bound state solutions of the $D$-dimensional Schrödinger equation for the Woods-Saxon potential
}

\author{
V. H. Badalov* \\ Institute for Physical Problems, Baku State University, \\ Z. Khalilov st. 23, AZ-1148, Baku, Azerbaijan
}

(Dated:)

\begin{abstract}
In this work, the analytical solutions of the $D$-dimensional Schrödinger equation are studied in great detail for the Wood-Saxon potential by taking advantage of the Pekeris approximation. Within a novel improved scheme to surmount centrifugal term, the energy eigenvalues and corresponding radial wave functions are found for any angular momentum case within the context of the Nikiforov-Uvarov (NU) and Supersymmetric quantum mechanics (SUSYQM) methods. In this way, based on these methods, the same expressions are obtained for the energy eigenvalues, and the expression of radial wave functions transformed each other is demonstrated. In addition, a finite number energy spectrum depending on the depth of the potential $V_{0}$, the radial $n_{r}$ and orbital $l$ quantum numbers and parameters $D, a, R_{0}$ are defined as well.
\end{abstract}

PACS numbers: 03.65.Ge, 03.65.-W, 03.65.Fd, 02.30.Gp

Keywords: Analytical solutions, Nikiforov - Uvarov method, Supersymmetric Quantum Mechanics, Pekeris approximation

${ }^{*}$ Electronic address: E-mail:badalovvatan@yahoo.com

Typeset by REVTEX 


\section{INTRODUCTION}

An analytical solution of the radial Schrödinger equation with a physical potential is of paramount importance in nonrelativistic quantum physics since the wave function and its associated eigenvalues contain all necessary information for full description of a quantum system. Along the years, there was a huge amount of research effort to solve exactly the radial Schrödinger equation for all values of $n_{r}$ and $l$ quantum numbers, but it could only be possible for a few specific potentials. In this way, there are several established analytical methods, including Polynomial solution [1 -3] ], Nikiforov - Uvarov method (NU) [4], Supersymmetric quantum mechanics method (SUSYQM) [5, 6], and Asymptotic iteration method (AIM) [7-13], to solve the radial Schrödinger equation exactly or quasi-exactly for $l \neq 0$ within these potentials. G.Levai et al. suggested a simple method for the proposed potentials for which the Schrödinger equation can be solved exactly with special functions [14] and presented relationship between the introduced formalism and SUSYQM [5]. Furthermore, in order to solve the Schrödinger equation applicable to problems of nonperturbative nature, P.Amore et al. introduced a novel method [15]. Thus, this method was applied to calculate the energies and wave functions of the ground and first excited state of the quantum anharmonic potential. It is well known that the Woods-Saxon potential [16] which we consider in the present work is one of the most realistic short-range potentials in physics. This potential plays a vital role in many branches of physics such as nuclear and particle, atomic, condensed matter, molecular and chemical physics. In fact, this potential cannot be solved exactly without using any approximation for $l \neq 0$ yet. However, S.Flügge gave an exact expression for the wave function and suggested a graphical method for the energy eigenvalues at $l=0$ [3].

In recent years, the NU [4] and SUSYQM [5, 6] methods with various approximations have been proposed for solving the Schrödinger equation analytically. Many papers show the power and simplicity of both of these methods in solving central and noncentral potentials [17-27]. The NU method is based on solving the second-order linear differential equation by reducing to a generalized equation of hypergeometric-type which is a secondorder type homogeneous differential equation with polynomials coefficients of degree not exceeding the corresponding order of differentiation, and SUSYQM method allows one to determine eigenstates of known analytically solvable potentials using algebraic operator for- 
malism without ever having to solve the Schrödinger differential equation by standard series technique. Hence it would be interesting and important to solve the nonrelativistic radial Schrödinger equation for Woods-Saxon potential for $l \neq 0$, since it has been extensively utilized to describe the bound and continuum states of the interacting systems. In this way, one can need to obtain the energy eigenvalues and corresponding eigenfunctions of the one particle problem within this potential. The NU method was used by C.Berkdemir et al. [28] solved the radial Schrödinger equation for the generalized Woods-Saxon potential for $l=0$. However, in this work, the authors made errors in application of the NU method, and these errors have led to incorrect results [29]. In the following works [30-34], authors made similar errors in application of the NU and AIM methods.

In our previous works [17-20], for the first time, we have given the comprehensive information of how to obtain analytically the exact energy eigenvalues and the corresponding wave functions of the radial Schrödinger and the radial Klein-Gordon equations with WoodsSaxon potential via NU method. In these works, the below approximation scheme - the Pekeris approximation [35] - was proposed for $V_{l}(r)=\frac{\hbar^{2} \tilde{l}(\tilde{l}+1)}{2 \mu r^{2}}$ the centrifugal potential in any arbitrary $l$ state:

$$
\frac{1}{r^{2}}=\frac{1}{R_{0}^{2}}\left(C_{0}+\frac{C_{1}}{1+e^{\frac{r-R_{0}}{a}}}+\frac{C_{2}}{\left(1+e^{\frac{r-R_{0}}{a}}\right)^{2}}\right),
$$

where $C_{0}, C_{1}, C_{2}$ quantities dependent on $R_{0}, a$ specific potential parameters were defined by comparing both sides of Eq.(1.1) expression expanding in the Taylor series around the point $r=R_{0}$. Furthermore, the D-dimensional Schrödinger equation with Woods-Saxon potential was solved within the context of the NU, AIM, and SUSYQM methods through the Pekeris approximation to the centrifugal potential, and the energy eigenvalues and corresponding radial wave functions are found for any arbitrary state as well [27].

In this study, the exact nonzero angular momentum solutions to $D$-dimensional Schrödinger equation with Woods-Saxon potential are presented within the context of the NU and SUSYQM methods. Since a nucleon in the interior of the nucleus feels a different potential than that near the surface of the nucleus, the study is extended there by using a new improved approximate scheme to deal with the centrifugal term, i.e., the Pekeris approximation is applied to the centrifugal potential $V_{l}(r)$ based on the settings $C_{0}, C_{1}, C_{2}$ quantities which are determined around the point $r=r_{\min }$ of the effective $l$ dependent 
potential $V_{\text {eff }}(r)$. Thus, the best part of this research work is that the same expressions are obtained for the energy eigenvalues and corresponding eigenfunctions in various values of $n_{r}$ and $l$ quantum numbers by taking advantage of the NU and SUSYQM methods.

This paper is structured as follows: After this introduction, the detailed description of the $D$-dimensional SE with the Woods-Saxon potential is given in Section II. Then in Section III, the impletemetion of NU method to $D$-dimensional SE is stated. In Section IV, the application of SUSYQM method to $D$-dimensional SE is presented. Next, the results and discussion are presented in Section V. Finally, the paper is concluded with brief summary in Section VI. Appendies A and B contain an overview of NU and SUSYQM methods respectively.

\section{THE $D$-DIMENSIONAL SCHRÖDINGER EQUATION WITH THE WOODS- SAXON POTENTIALS}

Woods and Saxon proposed a potential to describe the distribution of $20 \mathrm{MeV}$ protons on the heavy nuclei, such as platinum or nickel [16]. The spherical Woods-Saxon potential that was widely used to study the nuclear structure within the shell model has received significant attention in nuclear mean field model. The spherical standard Woods-Saxon potential [16] is defined by

$$
V(r)=-\frac{V_{0}}{1+e^{\frac{r-R_{0}}{a}}} \quad\left(a<<R_{0}\right) .
$$

This potential was also considered for description of interaction of a neutron with a heavy nucleus. The parameter $R_{0}$ represents the width of the nuclear radius; the parameter $a$ characterizes thickness of the superficial layer inside which the potential falls from value $V=0$ outside of a nucleus up to value $V=-V_{0}$ inside a nucleus. At $a=0$, one gets the simple potential well with jump of potential on the surface of a nucleus.

Using $D$-dimensional ( $D \geq 2$ ) polar coordinates with polar variable $r$ (hyperradius) and angular variables $r, \theta_{1}, \theta_{2}, \ldots, \theta_{D-2}, \phi$ (hyperangles), the Laplasian operator in polar coordinates $r, \theta_{1}, \theta_{2}, \ldots, \theta_{D-2}, \phi$ of $R^{D}$ is

$$
\nabla_{D}^{2}=r^{1-D} \frac{\partial}{\partial r}\left(r^{D-1} \frac{\partial}{\partial r}\right)+\frac{\Lambda_{D}^{2}}{r^{2}}
$$


where $\Lambda_{D}^{2}$ is a partial differential operator on the unit sphere $S^{D-1}$ (Laplace-Beltrami operator or grand orbital operator or hyperangular momentum operator) defined analogously to a three-dimensional (3D) angular momentum by Avery [36].

The $D$-dimensional Schrödinger equation with spherically symmetric potential $V(r)$ is of the form [36]

$$
\left(-\frac{\hbar^{2}}{2 \mu} \nabla_{D}^{2}+V(r)-E_{n_{r} l}\right) \psi_{n_{r} l m}\left(r, \Omega_{D}\right)=0,
$$

where $\mu$ is the reduced mass, $\hbar$ is the Planck's constant and

$$
\psi_{n_{r} l m}\left(r, \Omega_{D}\right)=R_{n_{r} l}(r) Y_{l m}\left(\Omega_{D}\right)
$$

The Laplasian operator divides into a hyper-radial part $r^{1-D} \frac{\partial}{\partial r}\left(r^{D-1} \frac{\partial}{\partial r}\right)$ and an angular part $\frac{\Lambda_{D}^{2}}{r^{2}}=-\frac{\hat{L}_{D}^{2}}{\hbar^{2} r^{2}}$ i.e.

$$
\nabla_{D}^{2}=r^{1-D} \frac{\partial}{\partial r}\left(r^{D-1} \frac{\partial}{\partial r}\right)-\frac{\hat{L}_{D}^{2}}{\hbar^{2} r^{2}}
$$

where $\hat{L}_{D}$ is the grand orbital angular momentum operator. The eigenfunctions of $\hat{L}_{D}^{2}$ are the hyper-spherical harmonics

$$
\hat{L}_{D}^{2} Y_{l m}\left(\Omega_{D}\right)=\hbar^{2} l(l+D-2) Y_{l m}\left(\Omega_{D}\right)
$$

where $l$ is the angular momentum quantum number.

After substituting Eqs.(2.4) - (2.6) into (2.3) and using $\psi_{n_{r} l m}\left(r, \Omega_{D}\right)$ as the eigenfunction of $\hat{L}_{D}^{2}$ with eigenvalue $\hbar^{2} l(l+D-2)$, we obtain an equation known as the hyper-radial Schrödinger equation with Woods-Saxon potential

$\frac{d^{2} R_{n_{r} l}(r)}{d r^{2}}+\frac{D-1}{r} \frac{d R_{n_{r} l}(r)}{d r}+\frac{2 \mu}{\hbar^{2}}\left[E_{n l}-V(r)-\frac{\hbar^{2} l(l+D-2)}{2 \mu r^{2}}\right] R_{n_{r} l}(r)=0,(0 \leq r<\infty)$.

Introducing a new function $u_{n_{r} l}(r)=r^{\frac{D-1}{2}} R_{n_{r} l}(r)$, Eq.(2.7) reduces

$$
\frac{d^{2} u_{n_{r} l}(r)}{d r^{2}}+\frac{2 \mu}{\hbar^{2}}\left[E_{n_{r} l}-V(r)-\frac{\hbar^{2}\left(l+\frac{D-1}{2}\right)\left(l+\frac{D-3}{2}\right)}{2 \mu r^{2}}\right] u_{n_{r} l}(r)=0,
$$

and introducing a new parametr $\tilde{l}=l+\frac{D-3}{2}$, Eq.(2.8) takes the form 


$$
\frac{d^{2} u_{n_{r} l}(r)}{d r^{2}}+\frac{2 \mu}{\hbar^{2}}\left[E_{n_{r} l}-V_{e f f}(r)\right] u_{n_{r} l}(r)=0
$$

where $V_{\text {eff }}(r)$ is effective potential, i.e.

$$
V_{e f f}(r)=V(r)+\frac{\hbar^{2} \tilde{l}(\tilde{l}+1)}{2 \mu r^{2}}
$$

Equation (2.9) has the same form as the equation for a particle in one dimension, except for two important differences. First, there is a repulsive effective potential proportional to the eigenvalue of $\hbar^{2} \tilde{l}(\tilde{l}+1)$. Second, the radial function must satisfy the boundary conditions $u(0)=0$ and $u(\infty)=0$.

It is well-known that the Schrödinger equation cannot be solved exactly for this potential at the value $l \neq 0$ by using the standard methods as SUSY and NU. From Eq.(2.10), it is seen that the effective potential is combination of the exponential and inverse square potentials which cannot be solved analytically. That is why, in order to solve this problem we can take the most widely used and convenient for our purposes Pekeris approximation. This approximation is based on the expansion the series for exponential cases depending on the internuclear distance of the centrifugal barrier, and there the terms up to second-order are considering.

After introducing the new variable $x=\frac{r-R_{0}}{R_{0}}$ or $r=R_{0}(1+x)$, the effective Woods-Saxon potential as following form:

$$
V_{e f f}(r)=-\frac{V_{0}}{1+e^{\alpha x}}+\frac{\tilde{\delta}}{(1+x)^{2}},
$$

where $\alpha=\frac{R_{0}}{a}$ and $\tilde{\delta}=\frac{\hbar^{2} \tilde{l}(\tilde{l}+1)}{2 \mu R_{0}^{2}}$. The extreme point of the effective potential $V_{\text {eff }}(r)$ is defined by the following equation

$$
\frac{\alpha V_{0} e^{\alpha x}}{\left(1+e^{\alpha x}\right)^{2}}=\frac{2 \tilde{\delta}}{(1+x)^{3}} .
$$

Since the solution of Eq.(2.12) depends on orbital $l$ quantum numbers, $x=x_{\min }=x_{l}$ $\left(r=r_{\min }=r_{l}\right)$.

Let us expand centrifugal potential $V_{l}(r)$ in Taylor series around the point of $x=x_{l}$ $\left(r=r_{l}\right)$ satisfied the transcendent Eq. (2.12):

$$
\begin{gathered}
V_{l}(r)=\frac{\hbar^{2} \tilde{l}(\tilde{l}+1)}{2 \mu r^{2}}=\frac{\hbar^{2} \tilde{l}(\tilde{l}+1)}{2 \mu R_{0}^{2}} \cdot \frac{\tilde{\delta}}{(1+x)^{2}}=\tilde{\delta}\left[\frac{1}{\left(1+x_{l}\right)^{2}}-\frac{2}{\left(1+x_{l}\right)^{3}} \cdot\left(x-x_{l}\right)+\right. \\
\left.+\frac{3}{\left(1+x_{l}\right)^{3}} \cdot\left(x-x_{l}\right)^{2}+o\left(\left(x-x_{l}\right)^{3}\right)\right]
\end{gathered} .
$$


According to the Pekeris approximation, $V_{l}(r)$ takes the form [17-20]

$$
\tilde{V}_{l}(r)=\tilde{\delta}\left(C_{0}+\frac{C_{1}}{1+e^{\alpha x}}+\frac{C_{2}}{\left(1+e^{\alpha x}\right)^{2}}\right)
$$

Let us expand the potential $\tilde{V}_{l}(r)$ in the Taylor series around the point of $x=x_{l} \quad\left(r=r_{l}\right)$ :

$$
\begin{aligned}
\tilde{V}_{l}(x) & =\tilde{\delta}\left[C_{0}+\frac{C_{1}}{1+e^{\alpha x_{l}}}+\frac{C_{2}}{\left(1+e^{\alpha x_{l}}\right)^{2}}-\left(\frac{\alpha C_{1} e^{\alpha x_{l}}}{\left(1+e^{\alpha x}\right)^{2}}+\frac{2 \alpha C_{2} e^{\alpha x_{l}}}{\left(1+e^{\alpha x_{l}}\right)^{3}}\right)\left(x-x_{l}\right)+\right. \\
& \left.-\left(\frac{\alpha^{2} C_{1} e^{\alpha x_{l}}\left(1-e^{\alpha x_{l}}\right)}{2\left(1+e^{\alpha x_{l}}\right)^{3}}+\frac{\alpha^{2} C_{2} e^{\alpha x_{l}}\left(1-2 e^{\alpha x_{l}}\right)}{\left(1+e^{\alpha x_{l}}\right)^{4}}\right)\left(x-x_{l}\right)^{2}+o\left(\left(x-x_{l}\right)^{3}\right)\right]
\end{aligned} .
$$

In order to define the constants $C_{0}, C_{1}$ and $C_{2}$, we compare the compatible degrees of same order of $x$ in Eqs.(2.13) and (2.15), and obtain the following algebraic system equations:

$$
\left\{\begin{array}{l}
C_{0}+\frac{C_{1}}{1+e^{\alpha x_{l}}}+\frac{C_{2}}{\left(1+e^{\alpha x_{l}}\right)^{2}}=\frac{1}{\left(1+x_{l}\right)^{2}} \\
\frac{\alpha C_{1} e^{\alpha x_{l}}}{\left(1+e^{\alpha x_{l}}\right)^{2}}+\frac{2 \alpha C_{2} e^{\alpha x_{l}}}{\left(1+e^{\alpha x_{l}}\right)^{3}}=\frac{2}{\left(1+x_{l}\right)^{3}} \\
\frac{\alpha^{2} C_{1} e^{\alpha x_{l}} l\left(1-e^{\alpha x_{l}}\right)}{2\left(1+e^{\alpha x_{l}}\right)^{3}}+\frac{\alpha^{2} C_{2} e^{\alpha x_{l}}\left(1-2 e^{\alpha x_{l}}\right)}{\left(1+e^{\alpha x_{l}}\right)^{4}}=-\frac{3}{\left(1+x_{l}\right)^{4}}
\end{array} .\right.
$$

From the solution of Eq.(2.16) algebraic system equations, for $C_{0}, C_{1}$ and $C_{2}$ constants, we get the following relations:

$$
\left\{\begin{array}{l}
C_{0}=\frac{1}{\left(1+x_{l}\right)^{2}}+\frac{\left(1+e^{\alpha x_{l}}\right)^{2}}{\alpha e^{\alpha x}\left(1+x_{l}\right)^{3}}\left[\frac{e^{-\alpha x_{l}-3}}{1+e^{\alpha x_{l}}}+\frac{3 e^{-\alpha x_{l}}}{\alpha\left(1+x_{l}\right)}\right] \\
C_{1}=\frac{2\left(1+e^{\alpha x_{l}}\right)^{2}}{\alpha e^{\alpha x_{l}}\left(1+x_{l}\right)^{3}}\left[2-e^{-\alpha x_{l}}-\frac{3\left(1+e^{-\alpha x_{l}}\right)}{\alpha\left(1+x_{l}\right)}\right] \\
C_{2}=\frac{\left(1+e^{\alpha x_{l}}\right)^{3}}{\alpha e^{\alpha x_{l}}\left(1+x_{l}\right)^{3}}\left[e^{-\alpha x_{l}}-1+\frac{3\left(1+e^{-\alpha x_{l}}\right)}{\alpha\left(1+x_{l}\right)}\right]
\end{array}\right.
$$

After Pekeris approximation, the effective potential as the following form:

$$
\tilde{V}_{e f f}(r)=K_{0}-\frac{K_{1}}{1+e^{\frac{r-R_{0}}{a}}}+\frac{K_{2}}{\left(1+e^{\frac{r-R_{0}}{a}}\right)^{2}},
$$

where $K_{0}=\tilde{\delta} C_{0}, K_{1}=V_{o}-\tilde{\delta} C_{1}, K_{2}=\tilde{\delta} C_{2}$, i.e.,

$$
\left\{\begin{array}{l}
K_{0}=\frac{\tilde{\delta}}{\left(1+x_{l}\right)^{2}}+\frac{\left(1+e^{\alpha x_{l}}\right)^{2} \tilde{\delta}}{\alpha e^{\alpha x}\left(1+x_{l}\right)^{3}}\left[\frac{e^{-\alpha x_{l}}-3}{1+e^{\alpha x} l}+\frac{3 e^{-\alpha x_{l}}}{\alpha\left(1+x_{l}\right)}\right] \\
K_{1}=V_{0}+\frac{2\left(1+e^{\alpha x_{l}}\right)^{2} \tilde{\delta}}{\alpha e^{\alpha x} l\left(1+x_{l}\right)^{3}}\left[e^{-\alpha x_{l}}-2+\frac{3\left(1+e^{-\alpha x_{l}}\right)}{\alpha\left(1+x_{l}\right)}\right] \\
K_{2}=\frac{\left(1+e^{\alpha x_{l}}\right)^{3} \tilde{\delta}}{\alpha e^{\alpha_{l}}\left(1+x_{l}\right)^{3}}\left[e^{-\alpha x_{l}}-1+\frac{3\left(1+e^{-\alpha x_{l}}\right)}{\alpha\left(1+x_{l}\right)}\right] .
\end{array}\right.
$$

If we consider $x_{l}=0$ in Eq.(2.17) relations, the constants $C_{0}, C_{1}$ and $C_{2}$ can be written in a closed form as $[17-20]$ :

$$
C_{0}=1-\frac{4}{\alpha}+\frac{12}{\alpha^{2}}, C_{1}=\frac{8}{\alpha}-\frac{48}{\alpha^{2}}, C_{2}=\frac{48}{\alpha^{2}}
$$


According to Eq.(2.12), Eq.(2.19) as the following form:

$$
\left\{\begin{array}{l}
K_{0}=\frac{V_{0}}{2}\left[\frac{\alpha\left(1+x_{l}\right)}{4 \cosh ^{2}\left(\frac{\alpha x_{l}}{2}\right)}+\frac{e^{-\alpha x_{l}-3}}{1+e^{\alpha x_{l}}}+\frac{3 e^{-\alpha x_{l}}}{\alpha\left(1+x_{l}\right)}\right] \\
K_{1}=V_{0}\left[e^{-\alpha x_{l}}-1+\frac{3\left(1+e^{\left.-\alpha x_{l}\right)}\right.}{\alpha\left(1+x_{l}\right)}\right] \\
K_{2}=V_{0}\left[\frac{6 \cosh ^{2}\left(\frac{\alpha x_{l}}{2}\right)}{\alpha\left(1+x_{l}\right)}-\sinh \left(\alpha x_{l}\right)\right]
\end{array}\right.
$$

It should be noted that this approximation preserves the original form of the effective $l$ dependent potential and is valid only for low vibrational energy cases.

Instead of solving the hyper-radial Schrödinger equation for the effective Woods-Saxon potential $V_{\text {eff }}(r)$ given by Eq.(2.11), we now solve the hyper-radial Schrödinger equation for

the new effective potential $\tilde{V}_{\text {eff }}(r)$ given by Eq.(2.18) obtained using the Pekeris approximation. Having inserted this new effective potential into Eq.(2.9), we obtain

$$
\frac{d^{2} u_{n_{r} l}(r)}{d r^{2}}+\frac{2 \mu}{\hbar^{2}}\left[E_{n_{r} l}-K_{0}+\frac{K_{1}}{1+e^{\frac{r-R_{0}}{a}}}-\frac{K_{2}}{\left(1+e^{\frac{r-R_{0}}{a}}\right)^{2}}\right] u_{n_{r} l}(r)=0
$$

If we rewrite equation Eq.(2.21) by using a new variable of the form $z=\left(1+e^{\frac{r-R_{0}}{a}}\right)^{-1}$, we obtain

$z^{2}(1-z)^{2} u^{\prime \prime}(z)+z(1-z)(1-2 z) u^{\prime}(z)+\frac{2 \mu a^{2}}{\hbar^{2}}\left[E-K_{0}+K_{1} z-K_{2} z^{2}\right] u(z)=0, \quad(0 \leq z \leq 1)$.

We use the following dimensionless notations

$$
\varepsilon^{2}=-\frac{2 \mu a^{2}\left(E-K_{0}\right)}{\hbar^{2}}>0, \beta^{2}=\frac{2 \mu a^{2} K_{1}}{\hbar^{2}}>0, \gamma^{2}=\frac{2 \mu a^{2} K_{2}}{\hbar^{2}}>0,
$$

we obtain

$$
u^{\prime \prime}(z)+\frac{1-2 z}{z(1-z)} u^{\prime}(z)+\frac{-\varepsilon^{2}+\beta^{2} z-\gamma^{2} z^{2}}{(z(1-z))^{2}} u(z)=0, \quad(0 \leq z \leq 1)
$$

with real $\varepsilon>0(E<0)$ for bound states; $\beta$ and $\gamma$ are real and positive.

\section{SOLUTION OF THE D-DIMENSIONAL SCHRÖDINGER EQUATION BY NIKIFOROV-UVAROV METHOD}

According to the NU-method from Eqs.(A.1) and (2.24), we obtain 


$$
\tilde{\tau}(z)=1-2 z ; \sigma(z)=z(1-z) ; \tilde{\sigma}(z)=-\varepsilon^{2}+\beta^{2} z-\gamma^{2} z^{2}
$$

and the new function $\pi(z)$ is

$$
\pi(z)= \pm \sqrt{\varepsilon^{2}+\left(k-\beta^{2}\right) z-\left(k-\gamma^{2}\right) z^{2}} .
$$

The constant parameter $k$ can be found employing the condition that the expression under the square root has a double zero, i.e., its discriminant is equal to zero. So, there are two possible functions for each $k$

$$
\pi(z)= \pm\left\{\begin{array}{l}
\left(\varepsilon-\sqrt{\varepsilon^{2}-\beta^{2}+\gamma^{2}}\right) z-\varepsilon, \text { for } k=\beta^{2}-2 \varepsilon^{2}+2 \varepsilon \sqrt{\varepsilon^{2}-\beta^{2}+\gamma^{2}} \\
\left(\varepsilon+\sqrt{\varepsilon^{2}-\beta^{2}+\gamma^{2}}\right) z-\varepsilon, \text { for } k=\beta^{2}-2 \varepsilon^{2}-2 \varepsilon \sqrt{\varepsilon^{2}-\beta^{2}+\gamma^{2}}
\end{array}\right.
$$

According to the NU-method, from the four possible forms of the polynomial $\pi(z)$ we select the one for which the function $\tau(z)$ has the negative derivative and root lies in the interval $(0,1)$. Therefore, the appropriate functions $\pi(z)$ and $\tau(z)$ have the following forms

$$
\begin{gathered}
\pi(z)=\varepsilon-\left(\varepsilon+\sqrt{\varepsilon^{2}-\beta^{2}+\gamma^{2}}\right) z \\
\tau(z)=1+2 \varepsilon-2\left(1+\varepsilon+\sqrt{\varepsilon^{2}-\beta^{2}+\gamma^{2}}\right) z,
\end{gathered}
$$

and

$$
k=\beta^{2}-2 \varepsilon^{2}-2 \varepsilon \sqrt{\varepsilon^{2}-\beta^{2}+\gamma^{2}} .
$$

Then, the constant $\lambda=k+\pi^{\prime}(z)$ is written as

$$
\lambda=\beta^{2}-2 \varepsilon^{2}-2 \varepsilon \sqrt{\varepsilon^{2}-\beta^{2}+\gamma^{2}}-\varepsilon-\sqrt{\varepsilon^{2}-\beta^{2}+\gamma^{2}} .
$$

An alternative definition of $\lambda_{n_{r}}$ (Eq.(A.9)) is

$$
\lambda=\lambda_{n_{r}}=2\left(\varepsilon+\sqrt{\varepsilon^{2}-\beta^{2}+\gamma^{2}}\right) n_{r}+n_{r}\left(n_{r}+1\right)
$$

Having compared Eq.(3.7) with Eq.(3.8)

$$
\beta^{2}-2 \varepsilon^{2}-2 \varepsilon \sqrt{\varepsilon^{2}-\beta^{2}+\gamma^{2}}-\varepsilon-\sqrt{\varepsilon^{2}-\beta^{2}+\gamma^{2}}=2\left(\varepsilon+\sqrt{\varepsilon^{2}-\beta^{2}+\gamma^{2}}\right) n_{r}+n_{r}\left(n_{r}+1\right),
$$


we obtain

$$
\varepsilon+\sqrt{\varepsilon^{2}-\beta^{2}+\gamma^{2}}+n_{r}+\frac{1}{2}-\frac{\sqrt{1+4 \gamma^{2}}}{2}=0
$$

or

$$
\varepsilon+\sqrt{\varepsilon^{2}-\beta^{2}+\gamma^{2}}-n^{\prime}=0 .
$$

Here

$$
n^{\prime}=-n_{r}+\frac{\sqrt{1+4 \gamma^{2}}-1}{2}
$$

and $n_{r}$ is the radial quantum number $\left(n_{r}=0,1,2, \ldots\right)$. From Eq.(3.11), we find

$$
\varepsilon=\frac{1}{2}\left(n^{\prime}+\frac{\beta^{2}-\gamma^{2}}{n^{\prime}}\right) .
$$

From the bound states $-V_{0}<E<0$ and finite wavefunction, we get $\varepsilon>0$ and $\sqrt{\varepsilon^{2}-\beta^{2}+\gamma^{2}}>0$, i.e. $n^{\prime}>0$ and $\left|\beta^{2}-\gamma^{2}\right|<n^{\prime 2}$. According to Eqs.(3.12) and (2.12) this relations can be recast into the form:

$$
\begin{gathered}
0 \leq n_{r}<\frac{1}{2}\left(\sqrt{1+\frac{8 \mu a^{2} K_{2}}{\hbar^{2}}}-1\right), \\
V_{0} R_{0}^{3} \geq \frac{4 \hbar^{2} \tilde{l}(\tilde{l}+1) a}{\mu} .
\end{gathered}
$$

Substituting the values of $\varepsilon, \beta, \gamma$ and $n^{\prime}$ into Eq.(3.13), one can find energy eigenvalues $E_{n_{r} l}^{(D)}$

$$
E_{n_{r} l}^{(D)}=K_{0}-\frac{K_{1}-K_{2}}{2}-\frac{\hbar^{2}}{32 \mu a^{2}}\left(\sqrt{1+\frac{8 \mu a^{2} K_{2}}{\hbar^{2}}}-2 n_{r}-1\right)^{2}-\frac{\frac{2 \mu a^{2}}{\hbar^{2}}\left(K_{1}-K_{2}\right)^{2}}{\left(\sqrt{1+\frac{8 \mu a^{2} K_{2}}{\hbar^{2}}}-2 n_{r}-1\right)^{2}} .
$$

If the conditions Eq.(3.14) and Eq.(3.15) are satisfied simultaneously, the bound states exist. Thus, the energy spectrum Eq.(3.16) is limited, i.e. we have only the finite number of energy eigenvalues.

For very large $V_{0}$, the $l$-dependent effective potential has the same form as the potential with $l=0$. When $D=3$, from Eq.(3.14) is seen that if $l=0$, then one gets $n_{r}<0$. 
Hence, the Schrödinger equation for the standard Woods-Saxon potential with zero angular momentum has no bound states. According to Eq.(3.16) the energy eigenvalues depend on the depth of the potential $V_{0}$, the width of the potential $R_{0}$, the thickness $a$ surface and $D$ parameter. Any energy eigenvalue must not be less than $-V_{0}$, i.e., $-V_{0}<E<0$. If constraints imposed on $n_{r}, V_{0}$ and $E$ satisfied, the bound states appear. From Eq.(3.15) is seen that the potential depth increases when the parameter $a$ increases, but the parameter $R_{0}$ is decreasing for given $l$ quantum number and vice versa. Therefore, one can say that the bound states exist within this potential.

In addition, we have seen that there are some restrictions on the potential parameters for the bound state solutions within the framework of quantum mechanics. Hence, when the values of the parameters $n_{r}, V_{0}$ and energy eigenvalues $E$ satisfy the conditions in Eqs.(3.14), (3.15) and $-V_{0}<E<0$ respectively, we obtain the bound states. We also point out that the exact results obtained for the standard Woods-Saxon potential may have some interesting applications for studying different quantum mechanical and nuclear scattering problems. Consequently, the found wave functions are physical ones.

Now, we are going to determine the radial eigenfunctions of this potential. Having substituted $\pi(z)$ and $\sigma(z)$ into Eq.(A.4) and then solving first-order differential equation, one can find the finite function $\Phi(z)$ in the interval $(0,1)$

$$
\Phi(z)=z^{\varepsilon}(1-z)^{\sqrt{\varepsilon^{2}-\beta^{2}+\gamma^{2}}} .
$$

It is easy to find the second part of the wave function from the definition of weight function

$$
\rho(z)=z^{2 \varepsilon}(1-z)^{2 \sqrt{\varepsilon^{2}-\beta^{2}+\gamma^{2}}}
$$

and substituting into Rodrigues relation Eq.(A.5), we get

$$
y_{n_{r}}(z)=B_{n_{r}} z^{-2 \varepsilon}(1-z)^{-2 \sqrt{\varepsilon^{2}-\beta^{2}+\gamma^{2}}} \frac{d^{n_{r}}}{d z^{n_{r}}}\left[z^{n_{r}+2 \varepsilon}(1-z)^{n_{r}+2 \sqrt{\varepsilon^{2}-\beta^{2}+\gamma^{2}}}\right],
$$

where $B_{n_{r}}$ is the normalization constant and its value is $\frac{1}{n_{r} !}$ [37]. Then, $y_{n_{r}}$ is given by the Jacobi polynomials

$$
y_{n_{r}}(z)=P_{n_{r}}^{\left(2 \varepsilon, 2 \sqrt{\varepsilon^{2}-\beta^{2}+\gamma^{2}}\right)}(1-2 z),
$$


where

$$
P_{n}^{(\alpha, \beta)}(1-2 z)=\frac{1}{n !} z^{-\alpha}(1-z)^{-\beta} \frac{d^{n}}{d z^{n}}\left[z^{n+\alpha}(1-z)^{n+\beta}\right] .
$$

The corresponding $u_{n_{r} l}(z)$ radial wave functions are found as:

$$
u_{n_{r} l}(z)=C_{n_{r} l} z^{\varepsilon}(1-z)^{\sqrt{\varepsilon^{2}-\beta^{2}+\gamma^{2}}} P_{n_{r}}^{\left(2 \varepsilon, 2 \sqrt{\varepsilon^{2}-\beta^{2}+\gamma^{2}}\right)}(1-2 z),
$$

where $C_{n_{r} l}$ is the normalization constant determined by using the following orthogonality relation:

$$
\int_{0}^{\infty}\left|R_{n_{r} l}(r)\right|^{2} r^{D-1} d r=\int_{0}^{\infty}\left|u_{n_{r} l}(r)\right|^{2} d r=a \int_{0}^{1} \frac{\left|u_{n_{r} l}(z)\right|^{2}}{z(1-z)} d z=1 .
$$

\section{SOLUTION OF THE D-DIMENSIONAL SCHRÖDINGER EQUATION BY SUPERSYMMETRIC QUANTUM MECHANICS METHOD}

According to SYSYQM, the eigenfunction of ground state $u_{0}(r)$ in Eq.(2.21) is a form as below

$$
u_{0}(r)=N \exp \left(-\frac{\sqrt{2 \mu}}{\hbar} \int W(r) d r\right),
$$

where $N$ is normalized constant and $W(r)$ is superpotential. The connection between the supersymmetric partner potentials $V_{1}(r)$ and $V_{2}(r)$ of the superpotential $W(r)$ is as follows [5]:

$$
V_{1}(r)=W^{2}(r)-\frac{\hbar}{\sqrt{2 \mu}} W^{\prime}(r)+E, \quad V_{2}(r)=W^{2}(r)+\frac{\hbar}{\sqrt{2 \mu}} W^{\prime}(r)+E .
$$

The particular solution of the Riccati equation Eq.(4.2) searches the following form:

$$
W(r)=-\frac{\hbar}{\sqrt{2 \mu}}\left(A+\frac{B}{1+e^{\frac{r-R_{0}}{a}}}\right),
$$

where $A$ and $B$ are unknown constants. Since $V_{1}(r)=\tilde{V}_{\text {eff }}(r)$, having inserted the relations Eqs.(2.18) and (4.3) into the expression Eq.(4.2), and from comparison of compatible quantities in the left and right sides of the equation, we find the following relations:

$$
A^{2}=-\frac{2 \mu}{\hbar^{2}}\left(E_{0}-K_{0}\right), \quad 2 A B-\frac{B}{a}=-\frac{2 \mu K_{1}}{\hbar^{2}}, \quad B^{2}+\frac{B}{a}=\frac{2 \mu K_{2}}{\hbar^{2}} .
$$


If we use Eqs.(2.23) for $A$ and $B$ parameters, Eqs.(4.4) are as follows:

$$
A^{2}=\frac{\varepsilon^{2}}{a^{2}}, 2 A B-\frac{B}{a}=-\frac{\beta^{2}}{a^{2}}, \quad B^{2}+\frac{B}{a}=\frac{\gamma^{2}}{a^{2}} .
$$

After inserting Eq.(4.3) into Eq.(4.1) and solving the integral, the eigenfunction for ground state is obtained as

$$
u_{0}(r)=N e^{A r}\left(1+e^{-\frac{r-R_{0}}{a}}\right)^{-a B} .
$$

$A$ must be less than zero, and $B$ must be greater than zero for the radial $u_{0}(r)$ wave function satisfy the boundary conditions $u_{0}(0)=0$ and $u_{0}(\infty)=0$. Under this circumstance, Eqs.(4.5) are as follows:

$$
\begin{gathered}
A=\frac{1}{2 a}-\frac{\beta^{2}}{a\left(\sqrt{1+4 \gamma^{2}}-1\right)}, \\
B=\frac{\sqrt{1+4 \gamma^{2}}-1}{2 a}, \\
E_{0}^{(D)}=K_{0}-\frac{\hbar^{2}}{2 \mu}\left[\frac{1}{2 a}-\frac{\beta^{2}}{a\left(\sqrt{1+4 \gamma^{2}}-1\right)}\right]^{2} .
\end{gathered}
$$

When $r \rightarrow \infty$, the chosen superpotential $W(r)$ is $W(r) \rightarrow-\frac{\hbar A}{\sqrt{2 \mu}}$. Having inserted Eq.(4.3) into Eq.(4.2), for supersymmetric partner potentials, we obtain:

$$
V_{1}(r)=\frac{\hbar^{2}}{2 \mu}\left[A^{2}+\frac{B^{2}+\frac{B}{a}}{\left(1+e^{\frac{r-R_{0}}{a}}\right)^{2}}+\frac{2 A B-\frac{B}{a}}{1+e^{\frac{r-R_{0}}{a}}}\right]
$$

and

$$
V_{2}(r)=\frac{\hbar^{2}}{2 \mu}\left[A^{2}+\frac{B^{2}-\frac{B}{a}}{\left(1+e^{\frac{r-R_{0}}{a}}\right)^{2}}+\frac{2 A B+\frac{B}{a}}{1+e^{\frac{r-R_{0}}{a}}}\right] .
$$

If we add side-by-side the second equation of Eqs.(4.5) to third equation of Eqs.(4.5), we obtain:

$$
2 A B+B^{2}=\frac{\gamma^{2}-\beta^{2}}{a^{2}}
$$

from here 


$$
A=\frac{\gamma^{2}-\beta^{2}}{2 a^{2} B}-\frac{B}{2}
$$

Two partner potentials $V_{1}(r)$ and $V_{2}(r)$ which differ from each other with additive constants and have the same functional form are called invariant potentials [38, 39]. Thus, for the partner potentials $V_{1}(r)$ and $V_{2}(r)$ given with Eqs.(4.10) and (4.11), the invariant forms are:

$$
\begin{gathered}
R\left(B_{1}\right)=V_{2}(B, r)-V_{1}\left(B_{1}, r\right)=-\frac{\hbar^{2}}{2 \mu}\left[\left(\frac{\gamma^{2}-\beta^{2}}{2 a^{2}\left(B-\frac{1}{a}\right)}-\frac{B-\frac{1}{a}}{2}\right)^{2}-\left(\frac{\gamma^{2}-\beta^{2}}{2 a^{2} B}-\frac{B}{2}\right)^{2}\right], \\
R\left(B_{i}\right)=V_{2}\left[B-\frac{i-1}{a}, r\right]-V_{1}\left[B-\frac{i}{a}, r\right]= \\
-\frac{\hbar^{2}}{2 \mu}\left[\left(\frac{\gamma^{2}-\beta^{2}}{2 a^{2}\left(B-\frac{i}{a}\right)}-\frac{B-\frac{i}{a}}{2}\right)^{2}-\left(\frac{\gamma^{2}-\beta^{2}}{2 a^{2}\left(B-\frac{i-1}{a}\right)}-\frac{B-\frac{i-1}{a}}{2}\right)^{2}\right]
\end{gathered}
$$

If we continue this procedure and make the substitution $B_{n_{r}}=B_{n_{r}-1}-\frac{1}{a}=B-\frac{n_{r}}{a}$ at every step until $B_{n_{r}} \geq 0$, the whole discrete spectrum of Hamiltonian $H_{-}(B)$ :

$$
\begin{gathered}
E_{n_{r} l}^{(D)}=E_{0}^{(D)}+\sum_{i=1}^{n_{r}} R\left(B_{i}\right), \\
E_{n_{r} l}^{(D)}=K_{0}-\frac{\hbar^{2}}{2 \mu}\left[\left(\frac{\gamma^{2}-\beta^{2}}{2 a^{2}\left(B-\frac{n_{r}}{a}\right)}-\frac{B-\frac{n_{r}}{a}}{2}\right)^{2}-\left(\frac{\gamma^{2}-\beta^{2}}{2 a^{2}\left(B-\frac{n_{r}-1}{a}\right)}-\frac{B-\frac{n_{r}-1}{a}}{2}\right)^{2}+\left(\frac{\gamma^{2}-\beta^{2}}{2 a^{2}\left(B-\frac{n_{r}-1}{a}\right)}-\frac{B-\frac{n_{r}-1}{a}}{2}\right)^{2}-\right. \\
-\left(\frac{\gamma^{2}-\beta^{2}}{2 a^{2}\left(B-\frac{n_{r}-2}{a}\right)}-\frac{B-\frac{n_{r}-2}{a}}{2}\right)^{2}+\ldots+\left(\frac{\gamma^{2}-\beta^{2}}{2 a^{2}\left(B-\frac{2}{a}\right)}-\frac{B-\frac{2}{a}}{2}\right)^{2}-\left(\frac{\gamma^{2}-\beta^{2}}{2 a^{2}\left(B-\frac{1}{a}\right)}-\frac{B-\frac{1}{a}}{2}\right)^{2}+ \\
\left.+\left(\frac{\gamma^{2}-\beta^{2}}{2 a^{2}\left(B-\frac{1}{a}\right)}-\frac{B-\frac{1}{a}}{2}\right)^{2}-\left(\frac{\gamma^{2}-\beta^{2}}{2 a^{2} B}-\frac{B}{2}\right)^{2}+\left(\frac{1}{2 a}-\frac{\beta^{2}}{a\left(\sqrt{1+4 \gamma^{2}}-1\right)}\right)^{2}\right]= \\
=K_{0}-\frac{\hbar^{2}}{2 \mu}\left[\frac{\gamma^{2}-\beta^{2}}{2 a^{2}\left(B-\frac{n_{r}}{a}\right)}-\frac{B-\frac{n_{r}}{a}}{2}\right]^{2}=K_{0}-\frac{\hbar^{2}}{2 \mu a^{2}}\left[\frac{\beta^{2}-\gamma^{2}}{\sqrt{1+4 \gamma^{2}}-2 n_{r}-1}+\frac{\sqrt{1+4 \gamma^{2}}-2 n_{r}-1}{4}\right]^{2} .
\end{gathered}
$$

Thereby, if we consider the parameter $\varepsilon, \beta, \gamma$ into Eq.(4.16), the obtained expression for energy eigenvalue in $l$-state will be same with expression Eq.(3.16) which was obtained by NU method. When $D=3$, there are no any bound states in system for $l=0$, because the inequalities $A<0, B>0$ are not satisfied. As a result, $n_{r}$ is less than zero and the calculated energy eigenvalues do not satisfy the inequality $-V_{0}<E<0$. It should be noted that the same conditions for $n_{r}$ and $V_{0}$ in Eq.(3.14) and Eq.(3.15) obtained by NU method 
are also determined from the following inequalities $B>0, A<0$. When $D>3$, there are bound states in system. It is seen from Eq.(3.16), the energy eigenvalue depends on the depth $V_{0}$ of the potential, the width $R_{0}$ potential, the thickness $a$ surface, and $D$ parameter. Thus, the determined conditions for $n_{r}, V_{0}$ and $E$, i.e., if the inequities $A<0, B>0$ and $-V_{0}<E<0$ are satisfied respectively, there are the bound states in the system, and the energy spectrum of these states is limited number. Based on Eqs.(B.15) and (B.17), the obtained result of radial Schrödinger equation by using the Eq.(4.6) of the ground state eigenfunction is exactly same with the result obtained by using NU method.

\section{RESULTS AND DISCUSSION}

In this chapter, in order to analyze the present qualitative findings, the single particle energy levels, the effective potentials and normalized wave functions of neutron moving under the average potential field of the ${ }^{56} \mathrm{Fe}$ nucleus are calculated for various $n_{r}$ and $l$ quantum numbers by using the empirical values $r_{0}=1.285 \mathrm{fm}$ and $a=0.65 \mathrm{fm}$ taken from Ref. [40]. Under these certain circumstances, the potential depth of mentioned potential is $V_{0}=(40.5+0.13 A) M e V=47.78 \mathrm{MeV}$, and the radius of the nucleus is $R_{0}=r_{0} A^{\frac{1}{3}}=$ $4.9162 \mathrm{fm}$. Here $A$ is the atomic mass number of ${ }^{56} \mathrm{Fe}$ nucleus. The reduced mass consists of neutron mass $m_{n}=1.00866 u$ and ${ }^{56} \mathrm{Fe}$ core mass with is $m_{A}=56 u$, and its value is $\mu=\frac{m_{A} \cdot m_{n}}{m_{A}+m_{n}}=0.990814 u$.

Calculated energies of the bound states and normalized wavefunction for $D=3$ in different values of $n_{r}$ and $l$ are presented in Table 1 indicate: when $1 \leq l \leq 4$ and $n_{r}=0$, there are bound states in system. However, when $1 \leq l \leq 4$ in $n_{r} \geq 1$ and $5 \leq l \leq 7$ in $n_{r}=0$ the energy values $E_{n_{r} l}$ are not satisfied the inequality $-V_{0} \leq E<0$, i.e., these findings cannot be considered physically and are only the mathematical results. Moreover, it is clearly seen from Fig. 1 that when $l=8$, the effective potential $V_{\text {eff }}(r)$ decreases monotonically, so there is no solution in transcendental equation i.e., there is neither physical nor mathematical result. Hence, there are no bound states in the system for the quantum numbers $5 \leq l \leq 7$. Furthermore, it can be clearly seen from Table I and Fig.1 that when the value of quantum number $l$ increases, the value of $r_{l}$ continues be closer the width of the nuclear radius $R_{0}$, and when $l=7$, the value of $r_{l}$ is greater than the width of the nuclear radius $R_{0}$. Namely, the

reason why there are no bound states in system for the quantum numbers $5 \leq l \leq 7$ could 
be due to the centrifugal potential expanding in the series around the surface of the nucleus. This fact is also confirmed by the result of Refs. [17-20] i.e., there are no bound states of system near the surface of the nucleus. In addition, the general behavior of normalization wave function for $1 \leq l \leq 4$ by a comparison with it for $5 \leq l \leq 7$ as shown in Fig.2 is very different. This alteration happened after $l=4$ which can be also related to the lack of bound states in system for $5 \leq l \leq 7$.

When $D=4$, the calculated energies of the bound states and normalized wavefunction in the different $n_{r}$ and $l$ values are presented in Table 2 . It is seen from Table 2 , there are bound states in system for $n_{r}=0$ and $1 \leq l \leq 3$. Hence, Eqs.(3.14) and (3.15) are satisfied for not only $l=0$ and $4 \leq l \leq 6$ in $n_{r} \geq 0$, but also $1 \leq l \leq 3$ in $n_{r} \geq 1$. However, the energy eigenvalues do not satisfy the inequality $-V_{0} \leq E<0$. It means that there are not the bound states in system for the mentioned $n_{r}$ radial and $l$ orbital quantum numbers. It should be noted that when $l \geq 7$, there is no solution in transcendental equation.

It seen from Table 1 and Table 2, the energy of the bound states increases with increasing of $D$ in the fixed same values of $n_{r}$ and $l$, i.e., $E_{01}^{(4)}>E_{01}^{(3)}, E_{02}^{(4)}>E_{02}^{(3)}, E_{03}^{(4)}>E_{03}^{(3)}$. It means that the repulsive force appears in system owing to the additional centrifugal potential $V_{l}(r)=\frac{\hbar^{2} \tilde{l}(\tilde{l}+1)}{2 \mu r^{2}}$. Therefore, in order to compensate this potential the energy of the bound state must increase [2, 41]. Note that when $D=3$ and $D=4$, there are not the bound states in system for $n_{r}=0, l=0$. Nevertheless, when $D=5$, there are the bound states in system for $n_{r}=0, l=0$ and its enegies is $E_{00}^{(5)}=-42.8980454 \mathrm{MeV}$. Thus, the reason why there are not the bound states in system for $n_{r}=0, l=0$ when $D=3$ and $D=4$ is related with the standard Woods-Saxon potential cannot describe the system fully. As a way out of this, the modified version of the standard Woods-Saxon potential such as the generalized Woods-Saxon potential and the spin and pseudospin symmetries in the standard WoodsSaxon potential can be utilized for solving the problem. Spin and pseudospin symmetries are symmetries of the Dirak Hamiltonian. Thus, pseudospin symmetry was discussed firstly in non-relativistic framework [42, 43], then in relativistic mean field theory. Comprehensive discussed in the Refs. [44-53] spin and pseudospin symmetries will be utilized for my further studies. 


\section{CONCLUSION}

To conclude, an analytical study of the D-dimensional space Schrödinger equation have been performed for Woods-Saxon potential using the improved approximation scheme to the centrifugal term for arbitrary $l$-states. There the energy eigenvalues of the bound states and corresponding eigenfunctions have been analytically found via both of NU and SUSYQM methods within the Pekeris approximation. The same expressions were obtained for the energy eigenvalues, and the expression of radial wavefunctions transformed each other was also shown by using these methods. The energy eigenvalues depending on $V_{0}, R_{0}, a$ and $D$ parameters have a finite number energy spectrum for standard Wood-Saxon potential, so it puts some restrictions on the potential parameters during the solution of related cases within the framework of quantum mechanics. In this way, if the potential parameters $V_{0}$, $n_{r}$ and energy eigenvalues $E$ satisfy the conditions in Eqs.(3.14), (3.15) and $-V_{0}<E<0$ respectively, it means there are bound states in system. It should be noted that the same limiting conditions were obtained for $V_{0}$ and $n_{r}$ thanks to both methods. Since there is the

practical interest for the energy spectrum in various potentials, investigating the features of eigenvalues is very important and actual with regard to arbitrary parameter of system. For illustration, the bound states energies of ${ }^{56} \mathrm{Fe}$ nucleus have been calculated and analyzed for some $l$ and $n_{r}$ values. The qualitative results of this study are expected to enable new possibilities for pure theoretical and experimental physicists, because the results are exact and more general.

[1] L.D. Landau and E.M. Lifshitz, Quantum Mechanics (Pergamon Press, London, 1958).

[2] W. Greiner, Quantum Mechanics, 4th. edn. (Springer, Berlin, 2001).

[3] S. Flügge, Practical Quantum Mechanics, Vol. 1 (Springer, Berlin, 1994).

[4] A.F. Nikiforov and V. B. Uvarov, Special Functions of Mathematical Physics (Birkhäuser, Basel, 1988).

[5] F. Cooper, A. Khare and U. Sukhatme, Phys. Rep. 251 (1995) 267.

[6] D.A. Morales, Chem. Phys. Letters, 394 (2004) 68.

[7] H. Ciftci, R.L. Hall and N. Saad, J. of Phys. : Math.Gen. 36 (2003) 11807.

[8] H. Ciftci, R.L. Hall and N. Saad, J. of Phys. : Math.Gen. 38 (2005) 1147. 
[9] H. Ciftci, R.L. Hall and N. Saad, Phys. Lett. A: 340 (2005) 388.

[10] O. Bayrak, I. Boztosun and H. ifti, Int. J. Quant. Chem. 107 (2007) 540.

[11] O. Bayrak and I. Boztosun, J. Phys. A 39 (2006) 6955.

[12] O. Bayrak, G. Kocak and I. Boztosun, J. Phys. A: Math. Gen. 39 (2006) 11521.

[13] H. Ciftci, O. Ozer and P. Roy, Chin. Phys. B 21 (2012) 010303.

[14] G. Levai and B.W. Williams, J. Phys. A: Math. Gen. 26 (1993) 3301.

[15] P. Amore, A. Aranda and A. De Pace, J. Phys. A: Math. Gen. 37 (2004) 3515.

[16] R.D. Woods and D.S. Saxon, Phys. Rev. 95 (1954) 577.

[17] V.H. Badalov, H.I. Ahmadov and S.V. Badalov, News Baku Univ., 2 (2008) 157.

[18] V.H. Badalov, H.I. Ahmadov, and A.I. Ahmadov, Int. J. Mod. Phys. E 18 (2009) 631.

[19] V.H. Badalov, H.I. Ahmadov, and S.V. Badalov, Int.J.Mod.Phys. E 19 (2010) 1463.

[20] V.H. Badalov, H.I. Ahmadov, math-ph/1111.4734.

[21] H.I.Ahmadov, C.Aydin, N.Sh.Huseynova, and O.Uzun. Int. J. Mod. Phys. E 22 (2013) 1350072 .

[22] A.I. Ahmadov, C. Aydin and O. Uzun. Int. J. Modern. Phys. A 29 (2014) 1450002.

[23] S.M. Ikhdair, R. Sever,Int. J. Theor. Phys. 46 (2007) 2384 (arXiv:quant-ph/0702186 V1).

[24] S.M. Ikhdair, R. Sever, Int. J. Mod. Phys. C 19 No. 9 (2008) 1425 (arXiv:quant-ph /0801.4857V3).

[25] S.M. Ikhdair, R. Sever, Int. J. Mod. Phys. C 19 No. 2 (2008) 221 (arXiv:quant-ph/0703008V1).

[26] S.M. Ikhdair, R. Sever, Int. J. Mod. Phys. $\quad$ E 17 No. 6 (2008) 1107 (arXiv:quant-ph/0605045V1).

[27] V.H. Badalov, J. Qafqaz Univ., 2 (2014) 178.

[28] C. Berkdemir, A. Berkdemir and R. Sever, Phys. Rev. C 72 (2005) 027001.

[29] Editorial Note: Polynomial solutions of the Schrodinger equation for the generalized WoodsSaxon potential [C. Berkdemir, A. Berkdemir and R. Sever, Phys. Rev. C 72 (2005) 027001; Phys. Rev. C 74 (2006) 039902(E)].

[30] S.M. Ikhdair and R. Sever, Ann. Phys. 16 (2007) 218 (arxiv: quant-ph/0610183 V1).

[31] A. Arda and R. Sever, Int.J.Mod.Phys. C 20 (2009) 651 (arxiv: math-ph/0901.2773 V1).

[32] A. Arda and R. Sever, Int.J.Mod.Phys. A 24(2009) 3985 (arxiv: quant-ph/0902.2008 V1).

[33] B.J. Falaye, M. Hamzavi and S.M. Ikhdair (arxiv: nucl-th/1207.1218 V1).

[34] S.M. Ikhdair, B.J. Falaye and M. Hamzavi, Chin. Phys. Lett. 30 (2013) 020305 (arxiv: quant- 
$\mathrm{ph} / 1307.8318$

[35] C.L. Pekeris, Phys. Rev. 45 (1934) 98.

[36] J. Avery, Hyperspherical Harmonics. Applications in Quantum Theory (Kluwer, Dordrecht, 1989).

[37] H. Bateman and A. Erdelyi, Higher Transcendental functions, Vol. 2. (McGraw-Hill, New York, 1953).

[38] L.E. Gendenshtein, JETP Lett.38, (1983) 356.

[39] L.E. Gendenshtein, I.V.Krive, Sov.Phys.Usp., 28 (1985) 645.

[40] C.M. Perey, F.G.Perey, J.K.Dickens and R.J. Silva, Phys.Rev. 175 (1968) 1460.

[41] L. E. Ballentine, Quantum Mechanics (World Scientic Publishing, Singapore, 1998).

[42] K.T. Hecht and A. Adler, Nucl.Phys. A 137 (1969) 129.

[43] A. Arima, M. Harvey and K. Shimizu, Phys. Lett. B 30 (1969) 517.

[44] H. Liang, J. Meng, S.-G. Zhou, Phys. Rep. 570 (2015) 1.

[45] J.N. Ginocchio, Phys. Rev. Lett. 78 (1997) 436.

[46] J. Meng, K. Sugawara-Tanabe, S. Yamaji, P. Ring and A. Arima, Phys. Rev. C 58 (1998) R628.

[47] J. Meng, K. Sugawara-Tanabe, S. Yamaji and A. Arima, Phys. Rev. C 59 (1999) 154.

[48] T.-S. Chen, H.-F. Lü, J. Meng, S.-Q. Zhang, S.-G. Zhou, Chin. Phys. Lett. 20 (2003) 358.

[49] S.-G. Zhou, J. Meng and P. Ring, Phys. Rev. Lett. 91 (2003) 262501.

[50] J.N. Ginocchio, Phys. Rep. 414 (2005) 165.

[51] J. Meng, H. Toki, S.-G. Zhou, S.-Q. Zhang, W.-H. Long, L.S. Geng, Prog. Part. Nucl. Phys. 57 (2006) 470.

[52] H. Liang, P. Zhao, Y. Zhang, J. Meng and N.V. Giai, Phys. Rev. C 83 (2011) 041301(R).

[53] H. Liang, S. Shen, P. Zhao and J. Meng, Phys. Rev. C 87 (2013) 014334. 


\begin{tabular}{|c|c|c|c|c|c|}
\hline$n_{r}$ & $l$ & $r_{l}, \mathrm{fm}$ & $V_{e f f, m i n}, \mathrm{MeV}$ & $E_{n_{r} l}, \mathrm{MeV}$ & $u_{n_{r} l}(z)$ \\
\hline 0 & 1 & 2.95578498158 & -40.71121848 & -42.8980494 & $7.419162631 z^{3.913357119}(1-z)^{0.2835207487}$ \\
\hline 1 & 1 & 2.95578498158 & -40.71121848 & -164.0083691 & Unbound \\
\hline 0 & 2 & 3.43967490298 & -32.59671725 & -30.9674480 & $4.630848265 z^{2.521449526}(1-z)^{0.1881862542}$ \\
\hline 1 & 2 & 3.43967490298 & -32.59671725 & -174.5240650 & Unbound \\
\hline 0 & 3 & 3.78599536866 & -22.94860534 & -18.3133413 & $3.248777890 z^{1.7924708185}(1-z)^{0.09698158616}$ \\
\hline 1 & 3 & 3.78599536866 & -22.94860534 & -209.1611062 & Unbound \\
\hline 0 & 4 & 4.07888427247 & -12.05092239 & -5.16198171 & $2.366451293 z^{1.293697429}(1-z)^{0.003675679733}$ \\
\hline 1 & 4 & 4.07888427247 & -12.05092239 & -385.5364626 & Unbound \\
\hline 0 & 5 & 4.35562101985 & -0.18380311 & 8.03190791 & $2.403325566 z^{0.895619899}(1-z)^{0.08910171673}$ \\
\hline 0 & 6 & 4.65152782641 & 12.32885320 & 20.44480441 & $2.284361438 z^{0.5317537204}(1-z)^{0.1866465558}$ \\
\hline 0 & 7 & 5.07501734690 & 24.95664294 & 20.79588752 & $2.835605734 z^{0.4259909093}(1-z)^{0.4933508522}$ \\
\hline
\end{tabular}

TABLE I: Calculated energies of the bound states and normalized wavefunction for $V_{0}=$ $47.78 \mathrm{MeV}, R_{0}=4.9162 \mathrm{fm}, a=0.65 \mathrm{fm}, D=3$ in different values of $n_{r}$ and $l$. 


\begin{tabular}{|c|c|c|c|c|c|}
\hline$n_{r}$ & $l$ & $r_{l}, \mathrm{fm}$ & $V_{\text {eff,min }}, \mathrm{MeV}$ & $E_{n_{r}}, \mathrm{MeV}$ & $u_{n_{r} l}(z)$ \\
\hline 0 & 0 & 2.56619312728 & -44.12161049 & -48.730119 & Unbound \\
\hline 1 & 0 & 2.56619312728 & -44.12161049 & -161.448867 & Unbound \\
\hline 0 & 1 & 3.22513008574 & -36.86366841 & -37.0225964 & $5.722788228 z^{3.074200941}(1-z)^{0.2347858502}$ \\
\hline 1 & 1 & 3.22513008574 & -36.86366841 & -167.8844225 & Unbound \\
\hline 0 & 2 & 3.62275083960 & -27.94626879 & -24.7231461 & $3.847953167 z^{2.114265381}(1-z)^{0.1424715417}$ \\
\hline 1 & 2 & 3.62275083960 & -27.94626879 & -186.4599846 & Unbound \\
\hline 0 & 3 & 3.93641236772 & -17.63870684 & -11.7764513 & $2.769256517 z^{1.525007303}(1-z)^{0.05127042688}$ \\
\hline 1 & 3 & 3.93641236772 & -17.63870684 & -257.2474288 & Unbound \\
\hline 0 & 4 & 4.21735772895 & -6.22020931 & 1.46781624 & $2.382992171 z^{1.0867462}(1-z)^{0.04134934644}$ \\
\hline 1 & 4 & 4.21735772895 & -6.22020931 & -1026.704467 & Unbound \\
\hline 0 & 5 & 4.49813167590 & 6.01629080 & 14.41696183 & $3.605399251 z^{0.7131560907}(1-z)^{0.5085916097}$ \\
\hline 0 & 6 & 4.82904585786 & 18.68343922 & 25.77233519 & $1.855453960 z^{0.3396915672}(1-z)^{0.1153903608}$ \\
\hline
\end{tabular}

TABLE II: Calculated energies of the bound states and normalized wavefunction for $V_{0}=$ $47.78 \mathrm{MeV}, R_{0}=4.9162 \mathrm{fm}, a=0.65 \mathrm{fm}, D=4$ in different values of $n_{r}$ and $l$. 


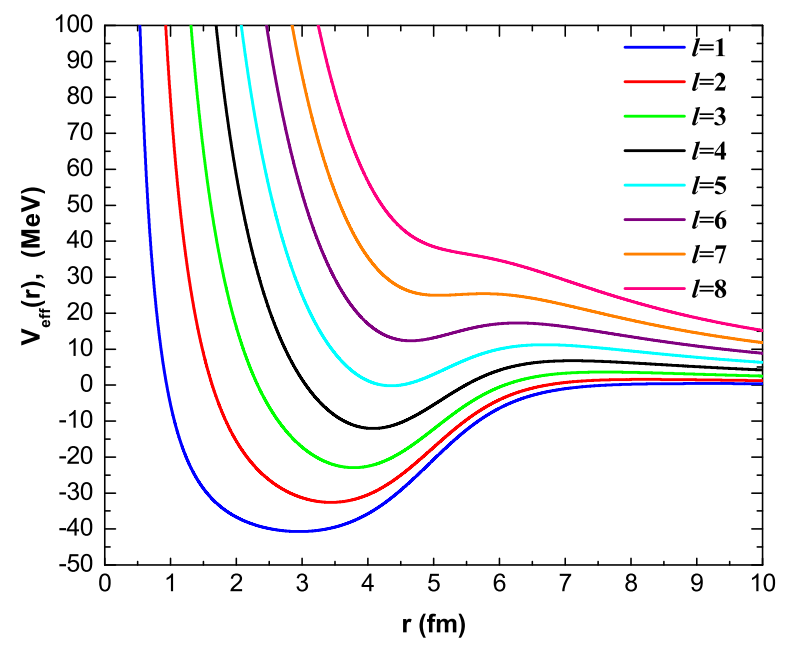

FIG. 1: The effective potential $V_{\text {eff }}(r)$ as a function of the internuclear $r$-distance and several $l$ quantum numbers for $V_{0}=47.78 \mathrm{MeV}, R_{0}=4.9162 \mathrm{fm}, a=0.65 \mathrm{fm}, \mathrm{D}=3$.

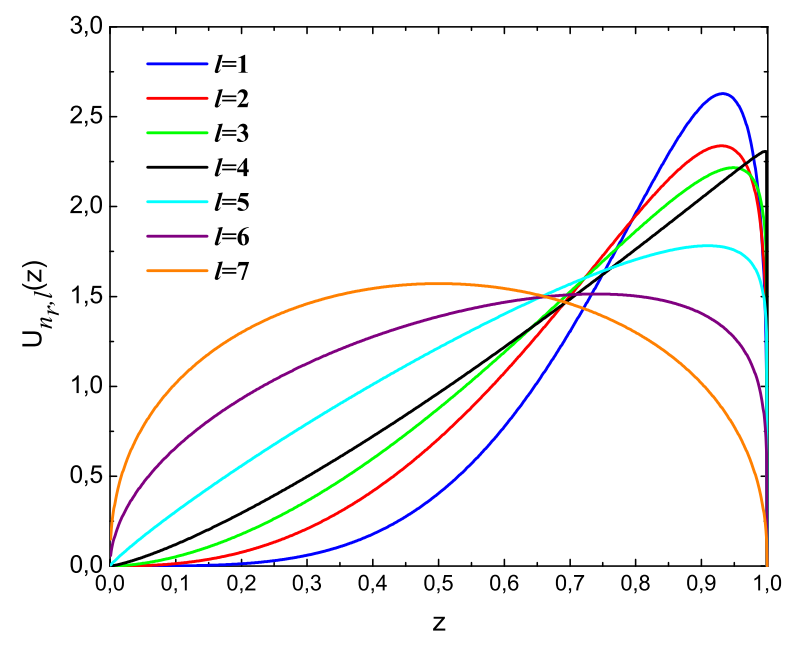

FIG. 2: The normalized wave function as a function of $z$ and several quantum numbers $l$ for $V_{0}=47.78 \mathrm{MeV}, R_{0}=4.9162 \mathrm{fm}, a=0.65 \mathrm{fm}, D=3, n_{r}=0$. 


\section{APPENDIX A. Nikiforov-Uvarov method}

The NU method is based on the solutions of general second order linear equations with special orthogonal functions. It has been extensively used to solve the non-relativistic Schrödinger equation and other Schrödinger-like equations. The one-dimensional (1D) Schrödinger equation or similar second-order differential equations can be written with NU method in the following form [4]:

$$
\psi^{\prime \prime}(z)+\frac{\tilde{\tau}(z)}{\sigma(z)} \psi^{\prime}(z)+\frac{\tilde{\sigma}(z)}{\sigma^{2}(z)} \psi(z)=0,
$$

where $\sigma(z)$ and $\tilde{\sigma}(z)$ are polynomials at most second-degree, and $\tilde{\tau}(z)$ is a first-degree polynomial.

By using in Eq.(A.1) the transformation

$$
\psi(z)=\Phi(z) y(z)
$$

one reduces it to the hypergeometric-type equation

$$
\sigma(z) y^{\prime \prime}+\tau(z) y^{\prime}+\lambda y=0 .
$$

The function $\Phi(z)$ is defined as the logarithmic derivative [4]

$$
\frac{\Phi^{\prime}(z)}{\Phi(z)}=\frac{\pi(z)}{\sigma(z)},
$$

where $\sigma(z)$ is at most the first-degree polynomial.

The another part of $\psi(z)$, namely $y(z)$ is the hypergeometric-type function, that for fixed $n$ is given by the Rodriguez relation

$$
y_{n}(z)=\frac{B_{n}}{\rho(z)} \frac{d^{n}}{d z^{n}}\left[\sigma^{n}(z) \rho(z)\right],
$$

where $B_{n}$ is the normalization constant and the weight function $\rho(z)$ must satisfy the condition [4]

$$
\frac{d}{d z}(\sigma(z) \rho(z))=\tau(z) \rho(z),
$$

with $\tau(z)=\tilde{\tau}(z)+2 \pi(z)$.

For accomplishment of the conditions imposed on function $\rho(z)$ the classical orthogonal polynomials, it is necessary, that polynomial $\tau(z)$ becomes equal to zero in some point of an interval $(a, b)$ and derivative of this polynomial for this interval at $\sigma(z)>0$ will be negative, i.e. $\tau^{\prime}(z)<0$.

[4]

The function $\pi(z)$ and the parameter $\lambda$ required for this method are defined as follows

$$
\begin{gathered}
\pi(z)=\frac{\sigma^{\prime}-\tilde{\tau}}{2} \pm \sqrt{\left(\frac{\sigma^{\prime}-\tilde{\tau}}{2}\right)^{2}-\tilde{\sigma}+k \sigma} \\
\lambda=k+\pi^{\prime}(z)
\end{gathered}
$$


On the other hand, in order to find the value of $k$, the expression under the square root must be the square of a polynomial. This is possible only if its discriminant is zero. Thus, the new eigenvalue equation for the Schrödinger equation becomes [4]

$$
\lambda=\lambda_{n}=-n \tau^{\prime}-\frac{n(n-1)}{2} \sigma^{\prime \prime}, \quad(n=0,1,2, \ldots) .
$$

After the comparison of Eq.(A.8) with Eq.(A.9), we obtain the energy eigenvalues.

\section{APPENDIX B. Supersymmetric Quantum Mechanics}

SUSYQM for $N=2$, we have two nilpotent operators, $Q$ and $Q^{+}$, satisfying the following algebra:

$$
\left\{Q, Q^{+}\right\}=H, \quad\{Q, Q\}=\left\{Q^{+}, Q^{+}\right\}=0
$$

where $H$ is the supersymmetric Hamiltonian, $Q=\left(\begin{array}{cc}0 & 0 \\ A^{-} & 0\end{array}\right)$ and $Q^{+}=\left(\begin{array}{cc}0 & A^{+} \\ 0 & 0\end{array}\right)$ are the operators of supercharges, $A^{-}$is bosonic operators and $A^{+}$is its adjoint. The supersymmetric $H$ Hamiltonian is given by [5]:

$$
H=\left(\begin{array}{cc}
A^{+} A^{-} & 0 \\
0 & A^{-} A^{+}
\end{array}\right)=\left(\begin{array}{cc}
H_{-} & 0 \\
0 & H_{+}
\end{array}\right)
$$

where $H_{-}$and $H_{+}$are called supersymmetric partner Hamiltonians. The supercharges $Q$ and $Q^{+}$commute with SUSY $H$ Hamiltonian: $[H, Q]=\left[H, Q^{+}\right]=0$.

If the ground state energy of a Hamiltonian $H$ is zero (i.e. $E_{0}=0$ ), it can always be written in a factorable form as a product of a pair of linear differential operators. That is why, one has from the Schrödinger equation that the ground state wave function $\psi_{o}(x)$ obeys

$$
H \psi_{o}(x)=-\frac{\hbar^{2}}{2 m} \frac{d^{2} \psi_{o}}{d x^{2}}+V(x) \psi_{o}(x)=0
$$

so that

$$
V(x)=\frac{\hbar^{2}}{2 m} \frac{\psi_{o}^{\prime \prime}(x)}{\psi_{o}(x)} .
$$

This allows a global reconstruction of the potential $V(x)$ from the knowledge of its ground state wave function which has no nodes. Once we realize this, it is now very simple to factorize the Hamiltonian using the following ansatz [5]:

$$
H_{-}=-\frac{\hbar^{2}}{2 m} \frac{d^{2}}{d x^{2}}+V(x)=A^{+} A^{-},
$$

where

$$
A^{-}=\frac{\hbar}{\sqrt{2 m}} \frac{d}{d x}+W(x), A^{+}=-\frac{\hbar}{\sqrt{2 m}} \frac{d}{d x}+W(x)
$$


By factorizing procedure of the Hamiltonian, the Riccati equation for Superpotential is obtained:

$$
V_{-}(x)=W^{2}(x)-\frac{\hbar}{\sqrt{2 m}} W^{\prime}(x) .
$$

The solution for $W(x)$ in terms of the ground state wave function is

$$
W(x)=-\frac{\hbar}{\sqrt{2 m}} \frac{\psi_{o}^{\prime}(x)}{\psi_{o}(x)} .
$$

This solution is obtained by recognizing that once we satisfy $A^{-} \psi_{0}(x)=0$, we automatically have a solution to $H \psi_{0}=A^{+} A^{-} \psi_{0}=0$.

The next step in constructing the SUSY theory related to the original Hamiltonian $H_{-}$is to define the operator $H_{+}=A^{-} A^{+}$obtained by reversing the order of $A^{-}$and $A^{+}$. A little simplification shows that the operator $H_{+}$is in fact a Hamiltonian corresponding to a new potential $V_{+}(x)$.

$$
H_{+}=-\frac{\hbar^{2}}{2 m} \frac{d^{2}}{d x^{2}}+V_{+}(x), \quad V_{+}(x)=W^{2}(x)+\frac{\hbar}{\sqrt{2 m}} W^{\prime}(x) .
$$

The potentials $V_{-}(x)$ and $V_{+}(x)$ are known as supersymmetric partner potentials. It is then clear that if the ground state energy of a Hamiltonian $H_{1}$ is $E_{(0)}^{(1)}$ with eigenfunction $\psi_{0}^{(1)}$ then in view of Eq.(B.5), it can always be written in the form below as,

$$
H_{1}=-\frac{\hbar^{2}}{2 m} \frac{d^{2}}{d x^{2}}+V_{1}(x)=A^{+} A^{-}+E_{0}^{(1)},
$$

where

$$
\begin{aligned}
& A_{1}^{-}=\frac{\hbar}{\sqrt{2 m}} \frac{d}{d x}+W_{1}(x), A_{1}^{+}=-\frac{\hbar}{\sqrt{2 m}} \frac{d}{d x}+W_{1}(x), \\
& V_{1}(x)=W_{1}^{2}(x)-\frac{\hbar}{\sqrt{2 m}} W_{1}^{\prime}(x)+E_{0}^{(1)}, \quad W_{\Pi}(x)=-\frac{\hbar}{\sqrt{2 m}} \frac{d \ln \psi_{0}^{(1)}}{d x} .
\end{aligned}
$$

The SUSY partner Hamiltonian is then given by [5]

$$
H_{2}=A_{1}^{-} A_{1}^{+}+E_{0}^{(1)}=-\frac{\hbar^{2}}{2 m} \frac{d^{2}}{d x^{2}}+V_{2}(x),
$$

where

$$
V_{2}(x)=W_{1}^{2}(x)+\frac{\hbar}{\sqrt{2 m}} W_{1}^{\prime}(x)+E_{0}^{(1)}=V_{1}(x)+\frac{2 \hbar}{\sqrt{2 m}} W_{1}^{\prime}(x)=V_{1}(x)-\frac{\hbar^{2}}{m} \frac{d^{2}}{d x^{2}}\left(\ln \psi_{0}^{(1)}\right) .
$$

From Eq.(B.12), the energy eigenvalues and eigenfunctions of the two Hamiltonians $H_{1}$ and $H_{2}$ are related by [5]

$$
E_{n}^{(2)}=E_{n+1}^{(1)}, \quad \psi_{n}^{(2)}=\left[E_{n+1}^{(1)}-E_{0}^{(1)}\right]^{-\frac{1}{2}} A_{1}^{-} \psi_{n+1}^{(1)}, \quad \psi_{n+1}^{(1)}=\left[E_{n}^{(2)}-E_{0}^{(1)}\right]^{-\frac{1}{2}} A_{1}^{+} \psi_{n}^{(2)} .
$$

Here $E_{n}^{(m)}$ is the energy level, where $n$ denotes the energy level and $(m)$ refers to the $m$ 'th Hamiltonian $H_{m}$.

In this way, it is clear that if the original Hamiltonian $H_{1}$ has $p \geq 1$ bound states with eigenvalues $E_{n}^{(1)}$, and eigenfunctions $\psi_{n}^{(1)}$ with $0<n<p$, then we can always generate a hierarchy of $(p-1)$ Hamiltonians $H_{2}, H_{3}, \ldots, H_{p}$ such that the $m$ 'th member of the hierarchy of Hamiltonians $\left(H_{m}\right)$ has the same eigenvalue spectrum as $H_{1}$ except that the 
first $(m-1)$ eigenvalues of $H_{1}$ are missing in $H_{m}$ [5] :

$$
H_{m}=A_{m}^{+} A_{m}^{-}+\mathrm{E}_{\mathrm{m}-1}^{(1)}=-\frac{\hbar^{2}}{2 m} \frac{d^{2}}{d x^{2}}+V_{m}(x)
$$

where

$$
A_{m}^{-}=\frac{\hbar}{\sqrt{2 m}} \frac{d}{d x}+W_{m}(x), \quad W_{m}(x)=-\frac{\hbar}{\sqrt{2 m}} \frac{d \ln \psi_{0}^{(m)}}{d x}, m=2,3,4, \cdots, p .
$$

One also has

$$
\begin{aligned}
& E_{n}^{(m)}=E_{n+1}^{(m-1)}=\cdots=E_{n+m-1}^{(1)}, \\
& \psi_{n}^{(m)}=\left[E_{n+m-1}^{(1)}-E_{m-2}^{(1)}\right]^{-\frac{1}{2}} \cdots\left[E_{n+m-1}^{(1)}-E_{0}^{(1)}\right]^{-\frac{1}{2}} A_{m-1}^{-} \cdots A_{1}^{-} \psi_{n+m-1}^{(1)}, \\
& V_{m}(x)=V_{1}(x)-\frac{\hbar^{2}}{m} \frac{d^{2}}{d x^{2}} \ln \left(\psi_{0}^{(1)} \cdots \psi_{0}^{(m-1)}\right) .
\end{aligned}
$$

i.e., knowing all the eigenvalues and eigenfunctions of $H_{1}$ we immediately know all the energy eigenvalues $E_{n}^{(1)}$ and eigenfunctions $\psi_{n}^{(1)}$ of the hierarchy of $(p-1)$ Hamiltonians $H_{2}, H_{3}, \ldots, H_{p}$. 